\title{
The Biomechanics of Spinal Deformity in Adolescent Idiopathic Scoliosis
}

\author{
Gregory S Van Blarcum ${ }^{1,2}$, Scott C Wagner ${ }^{1,2 *}$, Raymond M Meyer ${ }^{3}$, Daniel G Kang ${ }^{1,2}$ and Ronald A Lehman ${ }^{1,2}$ \\ ${ }^{1}$ Department of Orthopaedics, Walter Reed National Military Medical Center, Bethesda, USA \\ ${ }^{2}$ Division of Orthopaedics, Department of Surgery, Uniformed Services University of the Health Sciences, Bethesda, USA \\ ${ }^{3}$ Uniformed Services University of the Health Sciences, Bethesda, USA
}

\begin{abstract}
Given the variety of anatomic and physical variations that exist, the biomechanics of Adolescent Idiopathic Scoliosis (AIS) comprise a complex topic. Representing the fundamental mechanism behind the three-dimensional deformities observed in this condition, AIS develops as a result of these complex interactions between pathoanatomy and the developmental biomechanics of the spine. The progressive nature of the deformity relates to the interplay between bony anatomical alterations and the surrounding soft tissue response. Various classification systems have evolved to better understand AIS and provide guidance for its management, and myriad techniques have been developed to address the correction of spinal deformity in these patients. For appropriately selected patients, the treating surgeon must exploit all biomechanical advantages available $t$ counteract these deforming forces, and it is important that the biomechanics contributing to the progression of this condition be understood and corrected. All aspects of the case, including preoperative positioning and implant utilization, play a role in the amount of correction that can be obtained and maintained. These implants affect- and are affected by the forces present on the deformed spine, and manipulation of the surrounding anatomy may aid in obtaining the best surgical outcome.
\end{abstract}

\section{Introduction}

Spinal deformity biomechanics represent a complex topic, as many anatomic and physical variations exist that can affect the biomechanical behavior of the spinal column. Because the Homo sapiens species walks upright, the center of mass lies directly below the vertebral column, which stands in contrast to other vertebrate species $[1,2]$. In quadrupeds, the center of mass is typically anterior to the entirety of the thoracolumbar spine, and remains ventral to the base of the vertebral column [1,2]. In the human spine, because the center of mass lies directly below the vertebral column, axial compression remains the dominant force vector during ambulation, and variations in shear forces can be dramatic [3]. The normal anatomy of the human thoracolumbar spine has evolved as an adaptation to the resultant axial and bending forces, and the dorsally-directed shear forces that occur; the profound lumbar lordosis and associated thoracic kyphosis noted in normal human spinal anatomy is not observed in other vertebrates, including most closely-related primate species [3]. The anatomy of the human spine does not differ from other animals in the coronal plane as significantly as in the sagittal plane, and is less stable in rotation when placed under dorsally directed shear loading $[2,3]$. However, pathologic adaptations in functional spinal anatomy can develop, leading to major deformities that can significantly impact function, ambulation and quality of life. Adolescent Idiopathic Scoliosis (AIS) is a relatively common example, and the associated pathologic biomechanics-as well as the implications of such aberrant functional anatomy during surgical correction of AIS - are important to understand when considering treatment options for this condition.

\section{Development of Scoliosis}

Adolescent Idiopathic Scoliosis (AIS) is characterized by complex three-dimensional changes in the normal spinal anatomy, to include deformities in the axial, coronal and sagittal planes. As the deformity in AIS progresses, the vertebral column fails very predictably, and the stiffness of the vertebrae and the spinal column as a whole decreases in the direction of the major curvatures [2]. However, there is evidence that the etiology or progression of scoliosis is unrelated to the specific presence of coronal asymmetry [2]. Large epidemiological studies have shown that the sagittal plane deformities in the thoracic spine may precede development of coronal deformities, and may thus represent the most important structural changes inherent in progression of the deformity [2].
Growth progression has long been assumed to play a major role in the progression of AIS deformities. Growth of the anterior column has not been shown to be an element in the primary etiology of AIS, but likely does influence curvature protection and deformity acceleration [4]. A recent finite element analysis (FEA) study found the same correlation of anterior column growth and AIS progression when growth occurs in the presence of reduced stiffness of the intervertebral discs [5]. As the discs are deformed to a greater extent, it is reasonable to infer that their mechanical properties are unlikely to remain constant, and may therefore contribute to the actual progression of the curvature [5]. Curvature progression in AIS is related to the complex interplay between bony anatomy and the musculature surrounding the spine [6]. As a curve develops, the weight of the superior segments causes a lateral bending moment; it has been postulated that the minimal lateral offset of the apical vertebrae in a mild curve is around $2.5 \mathrm{~mm}$, which produces a moment of around $0.5 \mathrm{Nm}$ whenever the individual is standing upright [6]. Rueber et al. [7] proposed that it is an imbalance in the contraction forces of the spinal musculature to offset this bending moment that lead to progression of the curve. Others have postulated that it is the actual muscle imbalance and asymmetric contraction of the spinal musculature that leads to initial bending moments and further progression of curves [8]. Thus, while the exact relationship between the bending moment and the spinal musculature is unknown, it has been demonstrated in animal studies that when the spine is held fixed in lateral bent positions for several weeks, the tilts become semi-permanent [6]. This finding can likely be extrapolated into human models, and the longer the spine is held under

*Corresponding author: Scott C Wagner, Walter Reed National Military Medical Center, Department of Orthopaedics, 8901 Wisconsin Avenue, Bethesda, MD 20889, USA, Tel: (301)319-4818; Fax: (301)319-2361; E-mail: scott.cameron. wagner@gmail.com

Received June 18, 2014; Accepted December 06, 2014; Published December 08, 2014

Citation: Blarcum GSV, Wagner SC, Meyer RM, Kang DG, Lehman RA (2014) The Biomechanics of Spinal Deformity in Adolescent Idiopathic Scoliosis. J Spine 4: 197. doi:10.4172/2165-7939.1000197

Copyright: ( 2014 Blarcum GSV, et al. This is an open-access article distributed under the terms of the Creative Commons Attribution License, which permits unrestricted use, distribution, and reproduction in any medium, provided the original author and source are credited. 
these the deforming forces the more soft tissue creep and contracture is present, leading to structural permanence of the curve [6].

\section{Classification}

Classification systems for AIS have evolved over time, with more focus on the structure and flexibility of the curves as opposed to their location alone. Currently, AIS is classified based on the degree and location of major and minor curves in the coronal plane, as measured radiographically by the Cobb angle [9]. Ponseti first categorized curves based on location [10]. King expounded further on Ponseti's work, including not only the magnitude and location of the curves, but also making a distinctions based on the rigidity of the thoracic and lumbar curves [11]. The King classification was initially able to guide treatment strategies, including indications and levels needed for fusion [11], but this system was later shown to have poor inter- and intra- observer reliability [12]. The most widely used classification today was originally developed by Lenke [9] and defines curves as either structural (over 25 degrees on bending films) or non-structural, which is used to help guide treatment when fusion is indicated [9]. It is thus highly likely that the passive correction achievable is dependent on the flexibility of the deformity, the role the surrounding soft tissues play in the biomechanical maintenance of the curve cannot be understated [9].

However, there is also typically a significant rotational component to the deformity in AIS, which the Lenke classification does not completely address, and Nash and Moe [13] developed an early grading system for rotation based on the rotation of the pedicles seen on radiographs. More recently there has been the development of the Peking Union Medical College AIS classification, which takes into consideration the amount of apical vertebral rotation in addition to location and flexibility of the curves [14]. The guidelines for fusion are stricter than those as previously seen in the Lenke classification, taking into account the amount of rotation in addition to the flexibility of each curve [14]. Poncet et al. [15] categorized curves based primarily on curve torsion, making a distinction between unidirectional or bidirectional curve rotation as well as curve location, while others have subdivided curves based on the rotational component [16]. These classification systems incorporate modern advances in imaging modalities, particularly three dimensional reconstructions of the spine, to better appreciate and categorize the rotational component of AIS $[15,16]$. All of these systems highlight the complexity of the deformity and the convoluted nature of the force vectors imparted to the vertebral column during deformity progression.

\section{Deformity Correction}

If an AIS curve is indicated for surgical management, the physics of deformity correction must be understood to allow for adequate correction. Forces imparted on the spine during preoperative patient positioning can be exploited to improve the final degree of deformity correction [17]. Supine and prone preoperative positioning have been shown in a recent finite element analysis study to reduce the coronal deformity in AIS models [17]. Patient positioning has also been shown to have an effect on the curvature in other planes, particularly thoracic kyphosis and lumbar lordosis, while in the prone position [17]. The muscle relaxing effects of general anesthesia may contribute to the reduction of the deformity, indicating that asymmetric or otherwise modified preoperative positioning should be tailored to each patient's anatomy [17].

Utilizing a finite element model of the thoracolumbar spine, including the ribcage and sternum, Little et al. [18] showed that the degree of deformity correction achieved is directly proportional to the magnitude of corrective forces applied intra-operatively. The simulated intraoperative corrective forces were based upon previous, experimentally determined forces, and the FEA applied these forces in a manner consistent with the current standard of care for the reduction and instrumentation of AIS [18]. The authors noted that the most significant limitation to the degree of achievable correction was related primarily to direct contact between adjacent vertebral body endplates on the convex side of the deformity, while the greatest movement in the direction of curvature reduction was at the level of the apex and at the two immediately adjacent levels [18]. These findings are suggestive that the discrepancy between ideal deformity correction and achievable reduction is primarily affected by the vertebral anatomy of each individual patient [18].

While the magnitude of corrective forces applied intra-operatively is proportional to the degree of curvature reduction, the type and amount of instrumentation used to maintain deformity correction do not necessarily correlate with a greater degree of residual curvature reduction post-operatively [19]. Wang et al. [19] found no significant difference in final deformity correction when modeled with two separate instrumentation designs, despite significant differences in the amount of implants used. Additionally, in instrumentation designs with fewer pedicle screws, the average force at the bone-screw interface was significantly lower than in constructs with a higher number of pedicle screws [19]. However, the relationship between these findings and construct failure or clinical outcomes has not been has been elucidated in the literature. Martino et al. [20] more recently constructed a biomechanics analysis through case simulations. They ran the simulation using thirty-two different surgical variables, including the type of implant (density), number of levels undergoing derotation, the angle of derotation, and the force applied. They found that the implant type, density, and the vertebral derogation angle were the factors that most influenced correction [20]. Using bilateral, monoaxial pedicle screws with a derotation maneuver applied to three separate levels at the thoracic curve apex, maximal coronal and transverse plane correction was achieved [20]. The study concluded that it was possible to reduce the mean implant-vertebra forces by reducing the implant density by $50 \%$, and still maintain adequate correction [20].

Another recent study by Sun et al. [21] compared two different intraoperative correction techniques: vertebral column manipulation (VCM) and vertebral coplanar alignment (VCA) [21]. Vertebral column manipulation involves a utilizing a quadrilateral frame construct consisting of bilateral pedicle screws and rods to derotate the deformity, whereas VCA utilizes co-planar axes to correct the deformity [21]. Sun compared two groups of surgical patients matched for demographics and curve measurements using the two different techniques, finding a statistically significant difference in coronal plane correction; the VCM maneuver produced greater improvements in coronal alignment when compared to the VCA technique [21]. However all other planes showed no statistical difference, and neither group showed a significant loss of correction at follow-up [21].

Contouring of the rods used in the posterior construct can also have an effect on postoperative coronal and sagittal balance. Salmingo et al. [22] conducted a retrospective radiographic analysis of preoperative and postoperative implant rod geometry and angle of curvature for AIS patients undergoing deformity correction. The study found that rods inserted on the concave side of the deformity underwent statistically significant deformation after implantation, and a significant relationship between the degree of rod deformation and preoperative implant rod angle of curvature was demonstrated [22]. These results are suggestive that the concavity of the scoliotic deformity 
undergoes more significant biomechanical stresses than the convexity, and that the postoperative sagittal balance is significantly influenced by the preoperative contour of the rod used on the concave side of the curve [22]. The authors conclude that changes in the implant angle of curvature can cause under- or overcorrection of the deformity and special attention must be made to perfectly contouring the rod [22].

Release of the costo-vertebral joints via division the costo-vertebral and costo-transverse ligaments, as well as rib head resection, can reduce overall stiffness imparted to the spinal column and allow for improvements in deformity correction in the thoracic spine [23,24]. By uncoupling deflection on given thoracic levels from the deflection of the attached ribs, the surgeon can reduce the inherent biomechanical resistance of the torso to curvature correction [23]. However, the corrective advantage gained by releasing a costo-vertebral joint must be considered in the context of diminished stability of the thoracic spine, as well as increased strain in the ligamentous structures of the posterior elements [25]. Rib lengthening and shortening have also been utilized to improve coronal thoracic deformities, but convex-sided rib shortening or rib lengthening on the side of the concavity may induce significant and adverse force vectors that can oppose the reduction of the deformity [24]. Therefore, though the initial cosmetic effect of these techniques may be substantial, the induced loads may produce aberrant growth modulation that must be addressed [24].

\section{Conclusion}

Adolescent idiopathic scoliosis develops as a result of the complex interaction between pathoanatomy and the developmental biomechanics of the human spine. It is paramount that the forces contributing to the progression of this condition be understood and, in indicated patients, corrected, thereby maximizing patient functional outcomes and minimizing worsening of the deformity. Various techniques have been developed to address the correction of spinal deformity in these patients, and with continual advancements in medical technology - as well as improvements in the basic understanding of human functional spinal anatomy - surgical management strategies can continue to evolve.

\section{References}

1. Kouwenhoven JW, Vincken KL, Bartels LW, Meij BP, Oner FC, et al. (2006) Analysis of preexistent vertebral rotation in the normal quadruped spine. Spine (Phila Pa 1976) 31: E754-758.

2. Millner PA, Dickson RA (1996) Idiopathic scoliosis: biomechanics and biology. Eur Spine J 5: 362-373.

3. Kouwenhoven JW, Smit TH, Van der Veen AJ, Kingma I, van Dieen JH, et al (2007) Effects of dorsal versus ventral shear loads on the rotational stability of the thoracic spine: a biomechanical porcine and human cadaveric study. Spine 32: $2545-2550$

4. Shi L, Wang D, Driscoll M, Villemure I, Chu WC, et al. (2011) Biomechanical analysis and modeling of different vertebral growth patterns in adolescent idiopathic scoliosis and healthy subjects. Scoliosis 6: 11.

5. Drevelle X, Lafon Y, Ebermeyer E, Courtois I, Dubousset J, et al. (2010) Analysis of idiopathic scoliosis progression by using numerical simulation. Spine (Phila Pa 1976) 35: E407-412.
6. Schultz AB (1984) Biomechanical factors in the progression of idiopathic scoliosis. Ann Biomed Eng 12: 621-630.

7. Reuber M, Schultz A, McNeill T, Spencer D (1983) Trunk muscle myoelectric activities in idiopathic scoliosis. Spine (Phila Pa 1976) 8: 447-456.

8. Haderspeck K, Schultz A (1981) Progression of idiopathic scoliosis: an analysis of muscle actions and body weight influences. Spine (Phila Pa 1976) 6: 447455.

9. Lenke LG, Betz RR, Harms J, Bridwell KH, Clements DH, et al. (2001) Adolescent idiopathic scoliosis: a new classification to determine extent of spinal arthrodesis. J Bone Joint Surg Am 83-83A: 1169-81.

10. Ponseti IV, Friedman B (1950) Prognosis in idiopathic scoliosis. J Bone Joint Surg Am 32A: 381-395.

11. King HA, Moe JH, Bradford DS, Winter RB (1983) The selection of fusion levels in thoracic idiopathic scoliosis. J Bone Joint Surg Am 65: 1302-1313.

12. Lenke LG, Betz RR, Bridwell KH, Clements DH, Harms J, et al. (1998) Intraobserver and interobserver reliability of the classification of thoracic adolescent idiopathic scoliosis. J Bone Joint Surg Am 80: 1097-1106.

13. Nash CL Jr, Moe JH (1969) A study of vertebral rotation. J Bone Joint Surg Am 51: 223-229

14. Qiu G, Zhang J, Wang Y, Xu H, Zhang J, et al. (2005) A new operative classification of idiopathic scoliosis: a peking union medical college method. Spine (Phila Pa 1976) 30: 1419-1426.

15. Poncet P, Dansereau J, Labelle H (2001) Geometric torsion in idiopathic scoliosis: three-dimensional analysis and proposal for a new classification. Spine (Phila Pa 1976) 26: 2235-2243.

16. Sangole AP, Aubin CE, Labelle H (2009) Three-dimensional classification of thoracic scoliotic curves. Spine 34: 91-99.

7. Duke KA, Dansereau J, Labelle H (2005) Biomechanical simulations of scoliotic spine correction due to prone position and anaesthesia prior to surgical instrumentation. Clin Biomech 20: 923-931.

18. Little JP, Izatt MT, Labrom RD, Askin GN, Adam CJ (2013) An FE investigation simulating intra-operative corrective forces applied to correct scoliosis deformity. Scoliosis 8: 9 .

19. Wang X Aubin CE, Robitaille I, Labelle H (2012) Biomechanical comparison of alternative densities of pedicle screws for the treatment of adolescent idiopathic scoliosis. Eur Spine J 21: 1082-1090.

20. Martino J, Aubin CE, Labelle H, Wang X, Parent S, et al (2013) Biomechanica analysis of vertebral derotation techniques for the surgical correction of thoracic scoliosis. A numerical study through case simulations and a sensitivity analysis Spine 38: E73-83.

21. Sun L, Song Y, Liu L, An Y, Zhou C, Zhou Z, et al (2013) Bilateral apical vertebral derotation technique by vertebral column manipulation compared with vertebral coplanar alignment technique in the correction of Lenke type 1 idiopathic scoliosis. BMC Musculoskelet Disord 14: 175.

22. Salmingo RA, Tadano S, Abe Y I-M (2014) Influence of implant rod curvature on sagittal correction of scoliosis deformity. Spine J 14: 1432-1439.

23. Yao X, Blount TJ, Suzuki N, Brown LK, van der Walt CJ, et al. (2012) A biomechanical study on the effects of rib head release on thoracic spinal motion. Eur Spine J 21: 606-612.

24. Gréalou L, Aubin CE, Labelle H (2002) Rib cage surgery for the treatment of scoliosis: a biomechanical study of correction mechanisms. J Orthop Res 20: 1121-1128.

25. Little JP, Adam CJ (2011) Effects of surgical joint destabilization on load sharing between ligamentous structures in the thoracic spine: a finite element investigation. Clin Biomech (Bristol, Avon) 26: 895-903. 